\title{
Maternal or Infant Antiretroviral Drugs to Reduce HIV-1 Transmission
}

Charles S. Chasela, Ph.D., Michael G. Hudgens, Ph.D., Denise J. Jamieson, M.D., M.P.H., Dumbani Kayira, M.B., B.S., Mina C. Hosseinipour, M.D., M.P.H., Athena P. Kourtis, M.D., Ph.D., Francis Martinson, M.B., Ch.B., Ph.D., Gerald Tegha, B.Sc., Rodney J. Knight, Ph.D., Yusuf I. Ahmed, B.M., Deborah D. Kamwendo, M.Sc., Irving F. Hoffman, P.A., M.P.H., Sascha R. Ellington, M.S.P.H., Zebrone Kacheche, B.Sc., Alice Soko, R.N., Jeffrey B. Wiener, Ph.D., Susan A. Fiscus, Ph.D., Peter Kazembe, M.B., Ch.B., Innocent A. Mofolo, M.Sc., Maggie Chigwenembe, R.N., Dorothy S. Sichali, B.Sc., and Charles M. van der Horst, M.D. for the BAN Study Group*

University of North Carolina Project, Lilongwe, Malawi (C.S.C., D.K., M.C.H., F.M., G.T., D.D.K., Z.K., A.S., I.A.M., M.C., D.S.S.); the University of North Carolina (M.G.H., M.C.H., D.D.K., I.F.H., S.A.F., P.K., I.A.M., C.M.H.) and Principia (R.J.K.) - both in Chapel Hill; and the Centers for Disease Control and Prevention, Atlanta (D.J.J., A.P.K., Y.I.A., S.R.E., J.B.W.)

\section{Abstract}

Background-We evaluated the efficacy of a maternal triple-drug antiretroviral regimen or infant nevirapine prophylaxis for 28 weeks during breast-feeding to reduce postnatal transmission of human immunodeficiency virus type 1 (HIV-1) in Malawi.

\begin{abstract}
Methods-We randomly assigned 2369 HIV-1-positive, breast-feeding mothers with a CD4+ lymphocyte count of at least 250 cells per cubic millimeter and their infants to receive a maternal antiretroviral regimen, infant nevirapine, or no extended postnatal antiretroviral regimen (control group). All mothers and infants received perinatal prophylaxis with single-dose nevirapine and 1 week of zidovudine plus lamivudine. We used the Kaplan-Meier method to estimate the cumulative risk of HIV-1 transmission or death by 28 weeks among infants who were HIV-1negative 2 weeks after birth. Rates were compared with the use of the log-rank test.
\end{abstract}

Results-Among mother-infant pairs, 5.0\% of infants were HIV-1-positive at 2 weeks of life. The estimated risk of HIV-1 transmission between 2 and 28 weeks was higher in the control group $(5.7 \%)$ than in either the maternal-regimen group $(2.9 \%, \mathrm{P}=0.009)$ or the infant-regimen group $(1.7 \%, \mathrm{P}<0.001)$. The estimated risk of infant HIV-1 infection or death between 2 and 28 weeks was $7.0 \%$ in the control group, $4.1 \%$ in the maternal-regimen group ( $\mathrm{P}=0.02)$, and $2.6 \%$ in the infant-regimen group $(\mathrm{P}<0.001)$. The proportion of women with neutropenia was higher among those receiving the antiretroviral regimen (6.2\%) than among those in either the nevirapine group $(2.6 \%)$ or the control group (2.3\%). Among infants receiving nevirapine, $1.9 \%$ had a hypersensitivity reaction.

Copyright (C) 2010 Massachusetts Medical Society.

Address reprint requests to Dr. van der Horst at the Department of Medicine, University of North Carolina, CB \#3368, Chapel Hill, NC 27599-3368, or at cvdh@ med.unc.edu.

* Members of the Breastfeeding, Antiretrovirals, and Nutrition (BAN) study group are listed in the Appendix.

The findings and conclusions of this study are those of the authors and do not necessarily represent the official position of the Centers for Disease Control and Prevention.

No other potential conflict of interest relevant to this article was reported. Disclosure forms provided by the authors are available with the full text of this article at NEJM.org. 
Conclusions-The use of either a maternal antiretroviral regimen or infant nevirapine for 28 weeks was effective in reducing HIV-1 transmission during breast-feeding. (ClinicalTrials.gov number, NCT00164736.)

Approximately 200,000 infants worldwide become infected annually with human immunodeficiency virus type 1 (HIV-1) through breast-feeding. ${ }^{1,2}$ Without treatment, half of these infants will die before their second birthday. ${ }^{3}$ Although formula feeding decreases the risk of postnatal HIV transmission, it is associated with an increased rate of early death. ${ }^{4,5}$ Thus, exclusive breast-feeding is recommended by the World Health Organization (WHO) for infants of HIV-1-positive women for the first 6 months of life in resourcelimited settings. ${ }^{6}$

The use of antiretroviral drugs during pregnancy, labor, and delivery effectively reduces intrauterine and intrapartum HIV-1 transmission. ${ }^{7,8}$ However, without prophylaxis, an additional $9 \%$ of HIV-1-negative infants who are born to HIV-1-positive mothers will become infected after 18 months of breast-feeding. ${ }^{9}$ Several observational or uncontrolled studies that have examined the use of either an extended maternal triple-drug regimen or extended infant prophylaxis have suggested that these interventions reduce postnatal HIV-1 transmission. ${ }^{4,10-14}$ No studies to date have compared infant and maternal interventions. Two clinical trials have shown that infant prophylaxis with daily nevirapine for either 6 weeks or 14 weeks during breast-feeding reduced early postnatal HIV transmission. ${ }^{15,16}$

The Breastfeeding, Antiretrovirals, and Nutrition (BAN) study was a randomized, controlled trial evaluating the safety and efficacy of a maternal triple-drug antiretroviral regimen or infant nevirapine administered during 28 weeks of breast-feeding in reducing postpartum HIV transmission.

\section{Methods}

\section{Study Population}

The details of the methods that we used in this study have been described previously. ${ }^{17}$ Briefly, women were recruited at antenatal clinics in Lilongwe, Malawi, where HIV testing is conducted. ${ }^{18}$ Primary eligibility criteria included an age of at least 14 years, a gestation of 30 weeks or less, a CD4+ lymphocyte count of at least 250 cells per cubic millimeter ( $\geq 200$ cells per cubic millimeter before July 24, 2006), a hemoglobin level of at least $7 \mathrm{~g}$ per deciliter, an alanine aminotransferase level of no more than 2.5 times the upper limit of the normal range, and no serious complications of pregnancy. Women were excluded if they had a serious infection or reported previous use of antiretroviral drugs during this or previous pregnancies. After delivery, mother-infant pairs underwent randomization if they met secondary eligibility criteria: a birth weight of a least $2000 \mathrm{~g}$, no infant or maternal condition that would preclude the use of a study drug, the mother's acceptance of a 7-day maternal and infant perinatal antiretroviral regimen, and enrollment within 36 hours after delivery. Infants who were found to be HIV-1-positive by 2 weeks of life and their mothers were withdrawn from the study and were referred for care.

The study's protocol was approved by the Malawi National Health Science Research Committee and the institutional review boards at the University of North Carolina at Chapel Hill and the U.S. Centers for Disease Control and Prevention. All women provided written informed consent.

\section{Study Design and Interventions}

After delivery, according to a permuted-block method, mother-infant pairs were randomly assigned to a two-group nutritional intervention to promote maternal health and a three- 
group antiretroviral intervention - with drugs given to the mother (maternal-regimen group), infant (infant-regimen group), or neither the mother nor the infant (control group) to prevent HIV-1 transmission during breast-feeding. Since there was no interaction between the receipt of maternal nutritional supplementation and any of the antiviral interventions for the primary study outcomes (the rate of infant HIV-1 infection or the composite outcome of HIV-1 infection or death), this report focuses only on the antiretroviral intervention.

All mothers in labor and their newborn infants received a single dose of oral nevirapine. ${ }^{19}$ In addition, all mothers received zidovudine and lamivudine as a single tablet (Combivir, GlaxoSmithKline) containing $300 \mathrm{mg}$ of zidovudine and $150 \mathrm{mg}$ of lamivudine every 12 hours from the onset of labor to 7 days after birth. All infants also received twice-daily zidovudine ( $2 \mathrm{mg}$ per kilogram of body weight) and lamivudine (4 mg per kilogram) for 7 days.

All women were counseled to exclusively breastfeed followed by rapid weaning between 24 and 28 weeks postnatally. The interventions for both mothers and infants began after delivery and were continued until the cessation of breastfeeding but no longer than 28 weeks.

Women in the maternal-regimen group initially received Combivir twice daily and nevirapine at a dose of $200 \mathrm{mg}$ once daily for 2 weeks and twice daily thereafter until 28 weeks. After the first 39 women were randomly assigned to the maternal-regimen group, nevirapine was replaced with twice-daily nelfinavir (Viracept, Roche) at a dose of $1250 \mathrm{mg}$ for the next 146 women; nelfinavir was replaced with twice-daily lopinavir plus ritonavir (Kaletra, Abbott), a combination of $400 \mathrm{mg}$ of lopinavir and $100 \mathrm{mg}$ of ritonavir, for the remaining women. (The reason for this change was that in January 2005, the Food and Drug Administration issued a black-box warning concerning the use of nevirapine in women with a CD4+ lymphocyte count of more than 250 cells per cubic millimeter; at that time the study team began using the second-line regimen containing nelfinavir; for reasons of availability, safety, and potency, we then began using lopinavir plus ritonavir. ${ }^{17}$ ) Infants in the infantregimen group received a dose of nevirapine that increased according to age, ranging from $10 \mathrm{mg}$ daily in the first 2 weeks to $30 \mathrm{mg}$ daily for weeks 19 through 28 . Adherence was monitored by maternal self-report during five visits. Details regarding the management of toxic effects are provided in the Supplementary Appendix, available with the full text of this article at NEJM.org.

The study was conducted in accordance with the original protocol, except for the abovementioned replacement of nevirapine in the maternal-regimen group, the increase in the CD4+ count from 200 to 250 per cubic millimeter as an entry criterion, and the addition of trimethoprim-sulfamethoxazole prophylaxis for both mothers and infants. (Details are available in the trial protocol, available at NEJM.org.)

\section{Follow-up Visits and Study Procedures}

Mother-infant pairs were followed at 1, 2, 4, 6, 8, 12, 18, 21, 24, 28, 32, 36, 42, and 48 weeks after birth. A complete blood count and chemical analyses, including levels of alanine aminotransferase, were obtained from mothers and infants at delivery and at 2, 6, 12, 18, 24, 28,36 , and 48 weeks. In addition, specimens from mothers were obtained antenatally at screening and at weeks 4 and 8.

\section{Study End Points}

The primary efficacy end point was the rate of detection of HIV-1 infection at 28 weeks among infants who were uninfected at 2 weeks and among all infants who underwent randomization. Infants were tested for HIV-1 infection at birth and at 2, 12, 28, and 48 
weeks with the use of the Amplicor 1.5 DNA polymerase-chain-reaction (PCR) assay (Roche Molecular Systems). Positive specimens were confirmed by testing a specimen obtained at the next visit. If an infant was lost to follow-up or died before a confirmatory test was obtained, then a second specimen from the same day was tested at the reference laboratory at the University of North Carolina. Specimens with discrepant test results at the local laboratory were retested at the reference laboratory. Final infection status was determined by means of a review of test results by investigators who were unaware of studygroup assignments. The secondary efficacy end point was HIV-1-free survival by 28 weeks among infants who were uninfected at 2 weeks and among all infants who underwent randomization.

Clinical and laboratory adverse events were graded according to toxicity tables from the Division of AIDS at the National Institute of Allergy and Infectious Diseases (NIAID), as revised in March 2006. A separate grading system was used for infant neutropenia because of prevalent benign neutropenia among African infants, with a grade 3 toxicity defined as less than 400 cells per cubic millimeter. ${ }^{20}$

On the basis of the fourth interim analysis, according to O'Brien-Fleming stopping boundaries, the data and safety monitoring board for the Division of AIDS, which was overseeing the study, halted enrollment in the control group on March 26, 2008, after 668 of the planned 806 mother-infant pairs had been assigned to the control group. ${ }^{21}$

\section{Study Oversight}

GlaxoSmithKline, Abbott Laboratories, Boehringer Ingelheim, Roche Laboratories, and Bristol-Myers Squibb provided the medications used in this study. The manufacturers had no role in the design of the study, the collection or analysis of the data, or the decision to submit the manuscript for publication. All authors vouch for the completeness and accuracy of the data and the analyses.

\section{Statistical Analysis}

The Kaplan-Meier method was used to estimate the probability of each end point according to the age of the infants, and differences between the intervention groups and the control group were tested with the use of two-sided log-rank tests, stratified according to randomization to the nutritional-supplementation groups. At 6, 12, 18, and 28 weeks, a twosided $\mathrm{P}$ value was computed for the $\mathrm{z}$ statistic on the basis of the difference between the Kaplan-Meier estimates at that time point, with standard errors obtained with Greenwood's formula. The Cox proportional-hazards model was used to estimate hazard ratios and confidence intervals for the study outcomes comparing the two intervention groups with the control group, while adjusting for potentially prognostic baseline factors. Data regarding infants who did not reach a study end point by 28 weeks were censored at the last negative HIV-1 test or at 28 weeks, whichever occurred first. Data regarding mother-infant pairs who switched to an intervention group after enrollment was halted in the control group were censored at that time. To account for multiple comparisons with the control group, a $\mathrm{P}$ value of less than 0.025 was considered to indicate statistical significance.

\section{Results \\ Patients}

Of the 3572 HIV-1-positive pregnant women who consented to undergo study screening, $3109(87 \%)$ met the primary eligibility criteria. Of the 463 women (13\%) who were ineligible, 289 had a CD4+ count of less than either 200 cells or 250 cells per cubic millimeter (depending on the study protocol at the time of enrollment). Of the eligible 
women, 2791 (90\%) delivered an infant; 2382 mother-infant pairs met secondary eligibility criteria, although 13 declined to participate in the study, leaving 2369 mother-infant pairs to undergo randomization (Fig. 1).

Baseline demographic and laboratory characteristics of the 2369 mother-infant pairs who underwent randomization were well balanced among the three groups (Table 1). Loss to follow-up before 28 weeks occurred among $12 \%$ of mother-infant pairs in each study group.

On the basis of the mother's adherence report over five follow-up visits, infants received their prescribed nevirapine doses an average of $94 \%$ of the time, and mothers took their prescribed antiretroviral doses $89 \%$ of the time. The self-reported frequency of exclusive breast-feeding at 21 weeks after birth was $97 \%$ in the infant-nevirapine group and $96 \%$ in both the maternal-therapy group and the control group. By 28 weeks, complete cessation of breast-feeding was reported by 67 to $68 \%$ of mothers in each study group (Table 3 in the Supplementary Appendix).

\section{Infant HIV-1 Infection and Death}

By 2 weeks, infants in each of the three study groups had a similar estimated risk of infection: 5.4\% (95\% confidence interval [CI], 3.9 to 7.4) in the control group (reference group), $5.5 \%$ (95\% CI, 4.1 to $7.2 ; \mathrm{P}=0.97$ with the use of a $\mathrm{z}$ statistic) in the maternalregimen group, and $4.4 \%(95 \% \mathrm{CI}, 3.2$ to $6.0 ; \mathrm{P}=0.35)$ in the infant-regimen group. Among infants who were HIV-1-negative at 2 weeks, the estimated risk of HIV-1 infection by 28 weeks was $5.7 \%$ in the control group (reference group), $2.9 \%$ in the maternal-regimen group, and $1.7 \%$ in the infant-regimen group (Table 2 and Fig. 2A). Among infants who were HIV-1-negative at 2 weeks, there was a significant difference in the Kaplan-Meier estimates of HIV-1 infection beginning at 6 weeks between the infant-regimen group and the control group ( $\mathrm{P}=0.001$ with the use of a $\mathrm{z}$ statistic), whereas the difference between the control group and the maternal-regimen group reached borderline significance at 12 weeks $(P=0.04$ with the use of a $\mathrm{z}$ statistic $)$ and significance at 18 weeks $(P=0.01)$. In an analysis that included all infants who underwent randomization regardless of infection status at 2 weeks, the estimated risk of HIV-1 infection by 28 weeks was $10.9 \%$ (95\% CI, 8.7 to 13.6) in the control group, $8.2 \%$ (95\% CI, 6.5 to 10.3 ) in the maternal-regimen group, and $6.0 \%$ (95\% CI, 4.5 to 7.8 ) in the infant-regimen group (Fig. 2C).

Between randomization and week 28, there were 37 infant deaths, of which 8 were among HIV-1-positive infants. Six deaths among HIV-1-negative infants occurred less than 2 weeks after birth, a period that was not included in the primary analysis. Thus, there were 23 deaths among HIV-1-negative infants between weeks 2 and 28 (9 in the maternal-regimen group, 7 in the infant-regimen group, and 7 in the control group). The causes of death among all 37 infants were pneumonia (in 12 infants, of whom 5 were HIV-1-positive), sepsis (in 9 infants, of whom 1 was HIV-1-positive), meningitis (in 3 infants), diarrhea (in 2 infants), malaria (in 1 HIV-1-positive infant), unknown causes (in 6 infants, of whom 1 was HIV-1positive), and drowning, diabetes insipidus, organophosphate poisoning, and severe anemia presumably caused by trimethoprim-sulfamethoxazole toxicity in 1 infant each. (For details, see Table 6 in the Supplementary Appendix.)

Among infants who were HIV-1-negative at 2 weeks, the estimated risk of infection or death by 28 weeks was $7.0 \%$ in the control group (reference group), $4.1 \%$ in the maternalregimen group, and $2.6 \%$ in the infant-regimen group (Table 2 and Fig. 2B). Among all infants who underwent randomization, the proportion who had died or were HIV-1-positive by 28 weeks was $12.3 \%$ (95\% CI, 10.0 to 15.2) in the control group (reference group), 9.6\% (95\% CI, 7.7 to 11.8) in the maternal-regimen group, and 7.1\% (95\% CI, 5.5 to 9.1) in the infant-regimen group (Fig. 2D). Although the study was not designed to compare the two 
intervention groups, there was a borderline indication that the rate of HIV-1-negative survival among infants was greater when the infant received prophylaxis than when the mother received the antiretroviral regimen $(\mathrm{P}=0.07$ by the log-rank test) (Fig. 2D).

\section{Risk Factors for Infant HIV-1 infection}

Both the maternal and infant regimens remained significantly associated with a reduced risk of postnatal HIV-1 transmission after adjustment for maternal age and baseline CD4+ count among infants who were HIV-1-negative at 2 weeks (Table 3). Both interventions also remained associated with increased survival among HIV-1-negative infants. In addition, among all infants, nevirapine prophylaxis remained significantly associated with a reduced risk of postnatal HIV-1 transmission after multivariate adjustment for maternal age and baseline CD4+ count (adjusted hazard ratio, $0.54 ; 95 \%$ CI, 0.37 to 0.78 ); the association with the maternal regimen was of borderline significance (adjusted hazard ratio, $0.75 ; 95 \%$ CI, 0.53 to 1.05). Since the medications that were used for the maternal regimen changed over the course of the study, we examined the effect on transmission and HIV-1-negative survival according to the type of regimen. A log-rank test comparing the three maternal drug regimens suggested no difference in the rate of transmission by 28 weeks (for details, see the Supplementary Appendix).

\section{Serious Adverse Events}

Among mothers receiving the antiretroviral regimen, grade 3 or 4 neutropenia developed in $6.2 \%$, as compared with $2.6 \%$ in the infant-regimen group and $2.3 \%$ in the control group (Table 4). Nevirapine hypersensitivity developed in 6 of 39 women who received a nevirapine-based antiretroviral regimen ( 2 of whom had severe Stevens-Johnson syndrome). Among the infants receiving nevirapine, a hypersensitivity reaction developed in 16 within 4 weeks after the initiation of therapy; this reaction resolved after nevirapine was replaced with lamivudine. The hypersensitivity reactions in the infants consisted of rash and were accompanied by eosinophilia in 10 of the infants, with or without fever. A similar reaction developed in another infant whose mother was taking a nevirapine-containing antiretroviral regimen. There were no between-group differences in other measurements of toxic effects (Tables 4 and 5 in the Supplementary Appendix). The proportion of infants who had one or more nonfatal serious adverse events was $12.6 \%$ in the control group, $14.0 \%$ in the maternal-regimen group $(\mathrm{P}=0.59)$, and $15.7 \%$ in the infant-regimen group $(\mathrm{P}=0.15)$. The proportion of mothers who had one or more serious adverse events was $2.7 \%$ in the control group, $4.6 \%$ in the maternal-regimen group $(\mathrm{P}=0.05)$, and $1.6 \%$ in the infant-regimen group $(\mathrm{P}=0.41)$. There were two maternal deaths (from tuberculosis and hepatic necrosis) in the control group and none in the intervention groups.

\section{Discussion}

These data show that both a maternal antiretroviral regimen and infant nevirapine prophylaxis during 28 weeks of breast-feeding were effective in reducing postnatal HIV-1 transmission, as compared with a control regimen of a single dose of nevirapine and 7 days of zidovudine and lamivudine. The protective efficacy against HIV-1 transmission from 2 to 28 weeks was $74 \%$ for infant nevirapine and $53 \%$ for the maternal antiretroviral regimen. Infants also had significantly increased HIV-1-negative survival with both interventions, with a trend toward increased benefit when the drug regimen was administered directly to the infant.

Previous studies have shown that when an antiretroviral regimen was started during the antenatal period, rates of postnatal HIV-1 transmission of $1 \%$ or less by 6 months after birth were achieved. ${ }^{10,11,14}$ Results of the observational Kisumu Breastfeeding Study 
(ClinicalTrials.gov number, NCT00146380) showed a transmission rate of 2.8\% at 6 months. ${ }^{12}$ Our results suggest that the effect in the maternal-regimen group, which began 6 to 12 weeks after the effect in the infant-regimen group, could have reflected the time it took for the viral load to be suppressed sufficiently to prevent transmission. In order to achieve maximal reduction in HIV-1 transmission to the infant, the maternal regimen needs to start antenatally.

Other randomized, controlled trials have shown the efficacy of the administration of nevirapine to infants for the prevention of HIV-1 transmission during breast-feeding. In the Six-Week Extended-Dose Nevirapine (SWEN) study (NCT00061321), the infection rate among infants receiving 6 weeks of nevirapine was reduced from $9.0 \%$ to $6.9 \%$ at 6 months, as compared with infants receiving single-dose nevirapine. ${ }^{15}$ The Post-Exposure Prophylaxis of Infants (PEPI) study (NCT00115648) in Malawi showed a relative reduction of $60 \%$ (95\% CI, 42 to 73 ) in the rate of HIV-1 infection at 6 months among infants receiving 14 weeks of nevirapine, as compared with infants receiving single-dose nevirapine plus 1 week of zidovudine. ${ }^{16}$ Both the PEPI and SWEN studies included women with low CD4+ counts, and some mothers initiated an antiretroviral regimen during the study. The results of these studies suggest that an increased duration of nevirapine prophylaxis provides greater protection and that infants may benefit from daily nevirapine prophylaxis throughout breast-feeding without serious safety concerns. In an acknowledgment of the risk of weaning infants at 6 months, as shown in recent studies, ${ }^{22,23}$ the WHO has modified its recommendations to encourage breast-feeding for at least 12 months with more extended prophylaxis. ${ }^{6}$

An important aspect of our study was the inclusion of both maternal and infant regimens for the prevention of postnatal transmission. Infant nevirapine requires only a low-cost daily dose and is associated with manageable toxic effects. In addition, the potential for resistance is minimized, since a small proportion of infants receiving prophylaxis would become infected, and they are unlikely to transmit the virus. On the other hand, the maternal regimen may have advantages beyond simply protecting the infant, including maintaining maternal health. However, treatment interruption or poor adherence may increase the risk of resistant HIV-1 strains and restrict the mother's future treatment options. ${ }^{24}$ Furthermore, the maternal triple-drug regimen is costly and requires laboratory monitoring. ${ }^{25}$ Resistance may also occur in infants who become infected while their mothers are receiving the triple-drug regimen. ${ }^{26,27}$ Our finding of serious rashes in women with a high CD4+ count reinforces the need to avoid nevirapine-based regimens in these women.

The best way to protect maternal health is to increase HIV-1 testing of pregnant women and identify those in need of treatment. Women with a CD4+ count of less than 350 cells per cubic millimeter should start the antiretroviral regimen antenatally. For women who do not require treatment for their own health (i.e., those who have a CD4+ count of $>350$ cells per cubic millimeter), either the infant or maternal regimen can be used to prevent the transmission of HIV-1 in breast milk. In extremely resource-limited settings, nevirapine prophylaxis for the infant throughout breast-feeding may be the regimen of choice for reasons of cost, safety, and efficacy.

Health care providers and mothers can ultimately choose the option that best suits their cultural, economic, and individual needs, since there is now evidence for two effective options to prevent the transmission of HIV-1 to infants from their mothers during breastfeeding in resource-limited countries. 


\title{
Supplementary Material
}

Refer to Web version on PubMed Central for supplementary material.

\section{Acknowledgments}

\begin{abstract}
Supported by grants from the Prevention Research Centers Special Interest Project of the Centers for Disease Control and Prevention (SIP 13-01 U48-CCU409660-09 and SIP 26-04 U48-DP000059-01), the National Institute of Allergy and Infectious Diseases, the University of North Carolina Center for AIDS Research (P30-AI50410), the NIH Fogarty AIDS International Training and Research Program (DHHS/NIH/FIC 2-D43 Tw01039-06), Abbott Laboratories, GlaxoSmithKline, Boehringer Ingelheim, Roche Pharmaceuticals, Bristol-Myers Squibb, the Elizabeth Glaser Pediatric AIDS Foundation, the United Nations Children's Fund, the World Food Program, the Malawi Ministry of Health and Population, Johnson \& Johnson, and the U.S. Agency for International Development.
\end{abstract}

Dr. Van der Horst reports receiving grant support from Abbott Laboratories and GlaxoSmithKline; and Dr. Hosseinipour, receiving a lecture fee from Abbott Laboratories.

We thank all the women and their infants who participated in this study.

\section{Appendix}

The following investigators participated in this study as part of the BAN Study Team at the University of North Carolina Chapel Hill, the Centers for Disease Control and Prevention in Atlanta, and the UNC Project team in Lilongwe: L. Adair, Y. Ahmed, M. Ait-Khaled, S. Albrecht, S. Bangdiwala, R. Bayer, M. Bentley, B. Bramson, E. Bobrow, N. Boyle, S. Butera, C. Chasela, C. Chavula, J. Chimerang' ambe, M. Chigwenembe, M. Chikasema, N. Chikhungu, D. Chilongozi, G. Chiudzu, L. Chome, A. Cole, A. Corbett, A. Corneli, A. Cross, A. Duerr, H. Eliya, S. Ellington, J. Eron, S. Farr, Y. Owens Ferguson, S. Fiscus, S. Galvin, L. Guay, C. Heilig, I. Hoffman, E. Hooten, M. Hosseinipour, M. Hudgens, S. Hurst, L. Hyde, D. Jamieson, G. Joaki (deceased), D. Jones, Z. Kacheche, E. Kamanga, G. Kamanga, C. Kampani, P. Kamthunzi, D. Kamwendo, C. Kanyama, A. Kashuba, D. Kathyola, D. Kayira, P. Kazembe, C. King, R. Knight, A. Kourtis, R. Krysiak, J. Kumwenda, E. Loeliger, D. Long, M. Luhanga, V. Madhlopa, M. Majawa, A. Maida, C. Marcus, F. Martinson, C. Matiki (deceased), D. Mayers, I. Mayuni, M. McDonough, J. Meme, C. Merry, K. Mita, C. Mkomawanthu, G. Mndala, I. Mndala, A. Moses, A. Msika, W. Msungama, B. Mtimuni, J. Muita, N. Mumba, B. Musis, C. Mwansambo, G. Mwapasa, J. Nkhoma, R. Pendame, E. Piwoz, B. Raines, Z. Ramdas, J. Rublein, M. Ryan, I. Sanne, C. Sellers, D. Shugars, D. Sichali, S.-B. Smith, W. Snowden, A. Soko, A. Spensley, J.-M. Steens, G. Tegha, M. Tembo, R. Thomas, N. Thoofer, H.-C. Tien, B. Tohill, C. van der Horst, E. Waalberg, J. Wiener, C. Wilfert, P. Wiyo, I. Zgambo, and C. Zimba.

\section{REFERENCES}

1. Geneva: World Health Organization; 2007. HIV transmission through breastfeeding: a review of available evidence.

2. Geneva: Joint United Nations Programme on HIV/AIDS; 2007. AIDS epidemic update.

3. Newell ML, Coovadia H, Cortina-Borja M, Rollins N, Gaillard P, Dabis F. Mortality of infected and uninfected infants born to HIV-infected mothers in Africa: a pooled analysis. Lancet. 2004; 364:1236-1243. [PubMed: 15464184]

4. Thior I, Lockman S, Smeaton LM, et al. Breastfeeding plus infant zidovudine prophylaxis for 6 months vs formula feeding plus infant zidovudine for 1 month to reduce mother-to-child HIV transmission in Botswana: a randomized trial: the Mashi Study. JAMA. 2006; 296:794-805. [PubMed: 16905785] 
5. WHO Collaborative Study Team on the Role of Breastfeeding on the Prevention of Infant Mortality. Effect of breastfeeding on infant and child mortality due to infectious diseases in less developed countries: a pooled analysis. Lancet. 2000; 355:451-455. [PubMed: 10841125]

6. Geneva: World Health Organization; 2009. HIV and infant feeding: revised principles and recommendations: rapid advice. at http://www.who.int/child_adolescent_health/documents/9789241598873/en/index.html.)

7. Lallemant M, Jourdain G, Le Coeur S, et al. Single-dose nevirapine plus standard zidovudine to prevent mother-to-child transmission of HIV-1 in Thailand. N Engl J Med. 2004; 351:217-228. [PubMed: 15247338]

8. Achievements in public health: reduction in perinatal transmission of HIV infection - United States, 1985-2005. MMWR Morb Mortal Wkly Rep. 2006; 55:592-597. [PubMed: 16741495]

9. Coutsoudis A, Dabis F, Fawzi W, et al. Late postnatal transmission of HIV-1 in breast-fed children: an individual patient data meta-analysis. J Infect Dis. 2004; 189:2154-2166. [PubMed: 15181561]

10. Palombi L, Marazzi MC, Voetberg A, Magid NA. Treatment acceleration program and the experience of the DREAM program in prevention of mother-to-child transmission of HIV. AIDS. 2007; 21(Suppl 4):S65-S71. [PubMed: 17620755]

11. Shapiro, RL.; Hughes, M.; Ogwu, A., et al. A randomized trial comparing highly active antiretroviral therapy regimens for virologic efficacy and the prevention of mother-to-child HIV transmission among breastfeeding women in Botswana (The Mma Bana Study). Proceedings of the International AIDS Society Conference on HIV Pathogenesis, Treatment, and Prevention; July 19-22, 2009; Cape Town, South Africa. p. 175abstract.

12. Thomas, T.; Masaba, R.; Ndivo, R.; Zeh, C.; Borkowi, C. Prevention of mother-to-child transmission of HIV1 among breastfeeding mothers using HAART: the Kisumu breastfeeding study, Kisumu, Kenya, 2003-2007. Program and abstracts of the 15th Conference on Retroviruses and Opportunistic Infections; February 3-6, 2008; Boston. p. 87abstract.

13. Kilewo C, Karlsson K, Ngarina M, et al. Prevention of mother-to-child transmission of HIV-1 through breastfeeding by treating mothers with triple antiretroviral therapy in Dar es Salaam, Tanzania: the Mitra Plus study. J Acquir Immune Defic Syndr. 2009; 52:406-416. [PubMed: 19730269]

14. Peltier CA, Ndayisaba GF, Lepage $P$, et al. Breastfeeding with maternal antiretroviral therapy or formula feeding to prevent HIV postnatal mother-to-child transmission in Rwanda. AIDS. 2009; 23:2415-2423. [PubMed: 19730349]

15. Bedri A, Gudetta B, Isehak A, et al. Extended-dose nevirapine to 6 weeks of age for infants to prevent HIV transmission via breastfeeding in Ethiopia, India, and Uganda: an analysis of three randomised controlled trials. Lancet. 2008; 372:300-313. [PubMed: 18657709]

16. Kumwenda NI, Hoover DR, Mofenson LM, et al. Extended antiretroviral prophylaxis to reduce breast-milk HIV-1 transmission. N Engl J Med. 2008; 359:119-129. [PubMed: 18525035]

17. van der Horst C, Chasela C, Ahmed Y, et al. Modifications of a large HIV prevention clinical trial to fit changing realities: a case study of the Breastfeeding, Antiretroviral, and Nutrition (BAN) protocol in Lilongwe, Malawi. Contemp Clin Trials. 2009; 30:24-33. [PubMed: 18805510]

18. Moses A, Zimba C, Kamanga E, et al. Prevention of mother-to-child transmission: program changes and the effect on uptake of the HIVNET 012 regimen in Malawi. AIDS. 2008; 22:83-87. [PubMed: 18090395]

19. Guay LA, Musoke P, Fleming T, et al. Intrapartum and neonatal single-dose nevirapine compared with zidovudine for prevention of mother-to-child transmission of HIV-1 in Kampala, Uganda: HIV-NET 012 randomised trial. Lancet. 1999; 354:795-802. [PubMed: 10485720]

20. Kourtis AP, Bramson B, van der Horst C, et al. Low absolute neutrophil counts in African infants. J Int Assoc Physicians AIDS Care (Chic Ill). 2005; 4(3):73-76.

21. O'Brien PC, Fleming TR. A multiple testing procedure for clinical trials. Biometrics. 1979; 35:549-556. [PubMed: 497341]

22. Kafulafula G, Hoover DR, Taha TE, et al. Frequency of gastroenteritis and gastroenteritisassociated mortality with early weaning in HIV-1-uninfected children born to HIV-infected women in Malawi. J Acquir Immune Defic Syndr. 2010; 53:6-13. [PubMed: 19844183] 
23. Kuhn L, Aldrovandi GM, Sinkala M, et al. Effects of early, abrupt weaning on HIV-free survival of children in Zambia. N Engl J Med. 2008; 359:130-141. [PubMed: 18525036]

24. Darwich L, Esteve A, Ruiz L, Bellido R, Clotet B, Martinez-Picado J. Variability in the plasma concentration of efavirenz and nevirapine is associated with genotypic resistance after treatment interruption. Antivir Ther. 2008; 13:945-951. [PubMed: 19043929]

25. Hosseinipour MC, van Oosterhout JJ, Weigel R, et al. The public health approach to identify antiretroviral therapy failure: high-level nucleoside reverse transcriptase inhibitor resistance among Malawians failing first-line antiretroviral therapy. AIDS. 2009; 23:1127-1134. [PubMed: 19417582]

26. Zeh, C.; Weidle, P.; Nafisa, L.; Musuluma, H.; Okonji, J.; Anyango, E. Emergence of HIV-1 drug resistance among breastfeeding infants born to HIV-infected mothers taking antiretrovirals for prevention of mother-to-child transmission of HIV: the Kisumu Breastfeeding Study, Kenya. Program and abstracts of the 15th Conference on Retroviruses and Opportunistic Infections; February 3-6, 2008; Boston. p. 102abstract.

27. Lidstrom, J.; Guay, L.; Musoke, P., et al. Multi-class drug resistance arises frequently in HIVinfected breastfeeding infants whose mothers initiate HAART postpartum. Program and abstracts of the 17th Conference on Retroviruses and Opportunistic Infections; February 16-19, 2010; San Francisco. p. 433abstract. 


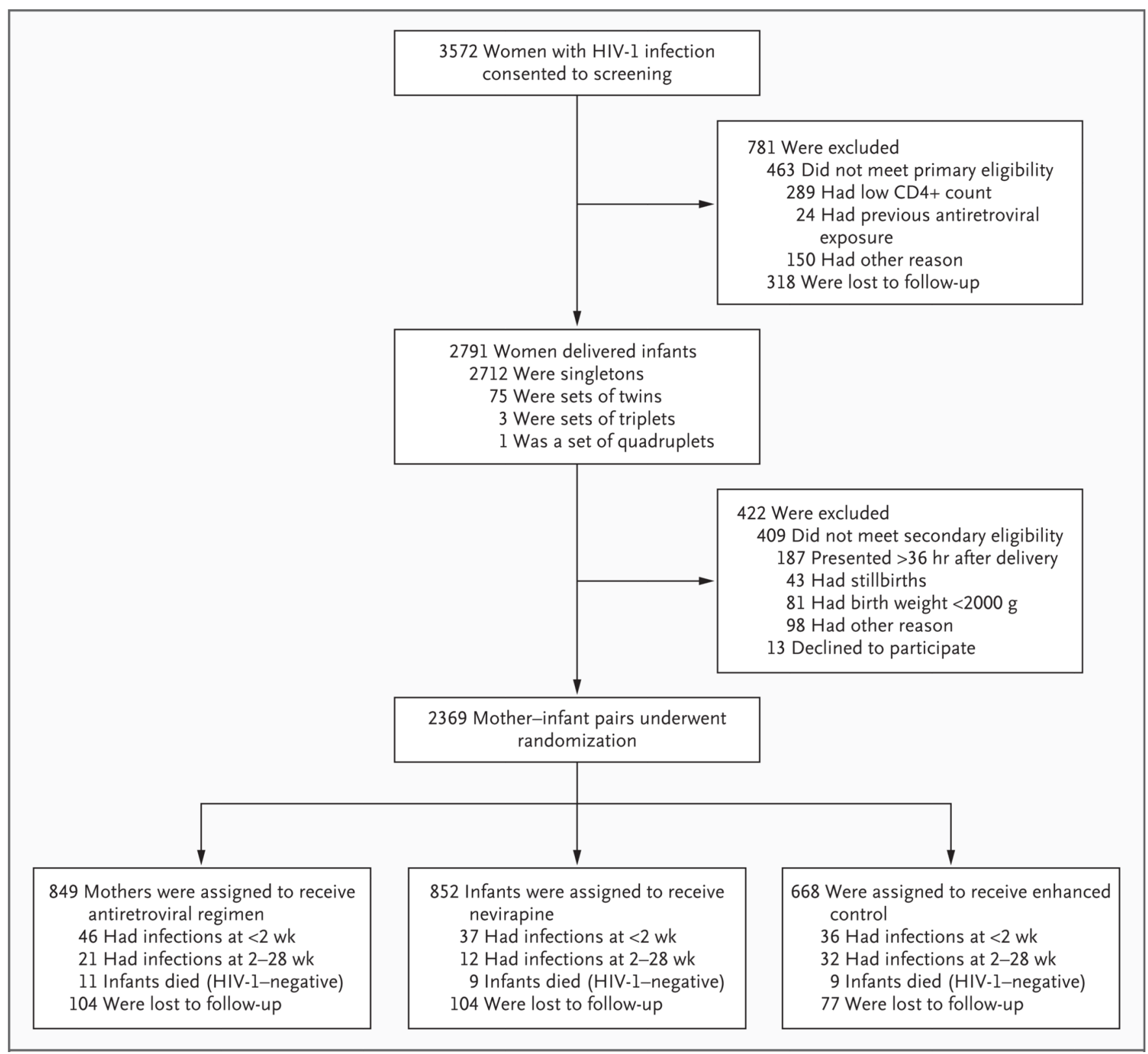

Figure 1. Enrollment and Outcomes

On March 26, 2008, the data and safety monitoring board stopped enrollment in the control group after 668 of the planned 806 mother-infant pairs had been assigned to that group.

Hence, the numbers of patients who were enrolled in the three study groups were uneven. Of the 29 infants who died during the study from non-HIV-1 causes, each had had a negative test for HIV-1 before death. 


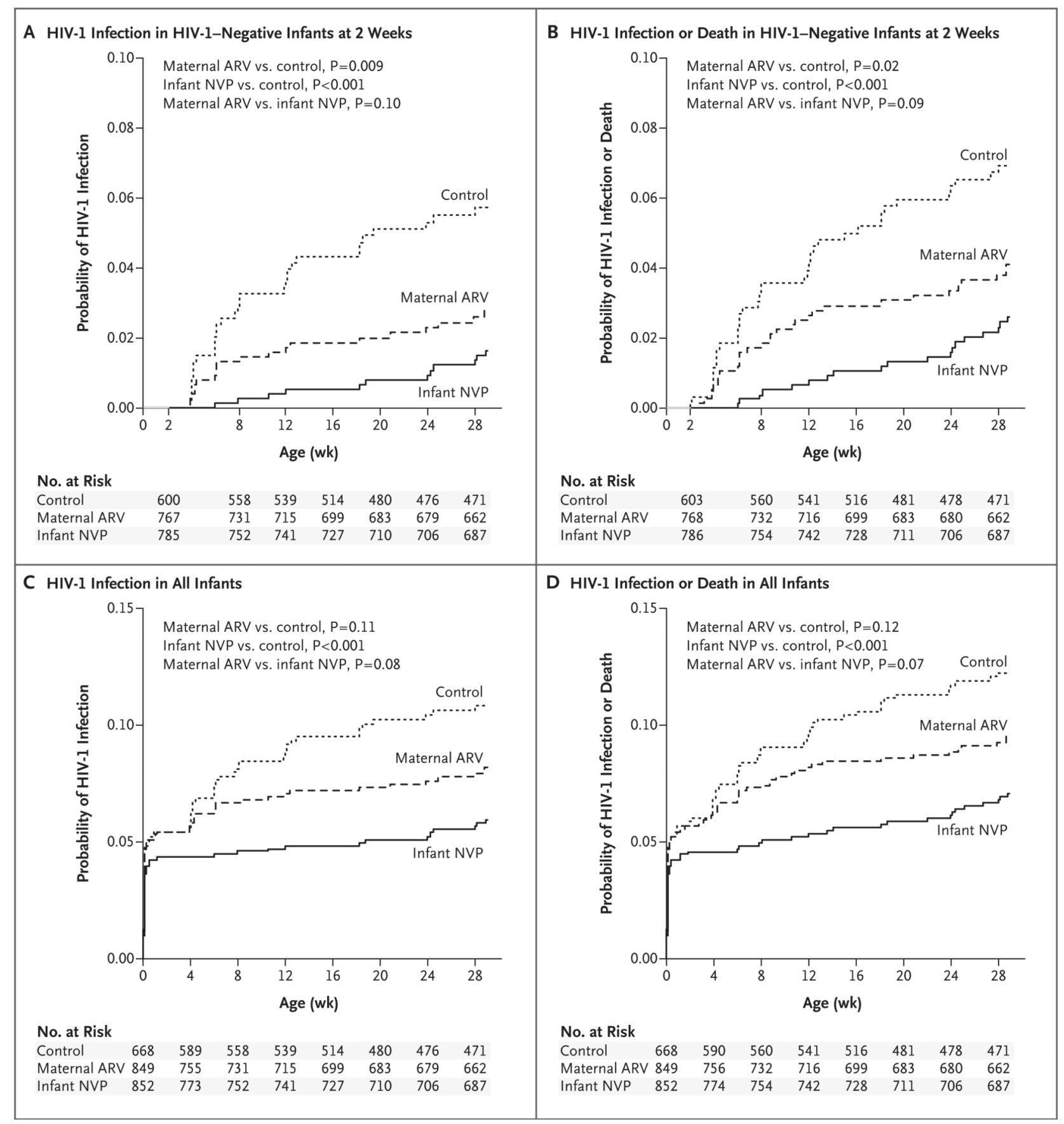

Figure 2. Kaplan-Meier Estimates of the Cumulative Risk of Infant HIV-1 Infection or a Composite of HIV-1 Infection or Death by 28 Weeks

Shown is the probability of HIV-1 infection or a composite of HIV-1 infection or death among infants who tested negative for HIV-1 infection at 2 weeks (Panels A and B) and among all infants who underwent randomization (Panels $\mathrm{C}$ and D) in three groups of mother-infants pairs: women who received an antiretroviral (ARV) regimen, infants who received nevirapine (NVP) prophylaxis, and control subjects. Rates were compared with the use of the log-rank test. 


\section{Table 1}

Baseline Characteristics of 2369 Mother-Infant Pairs.

\begin{tabular}{|c|c|c|c|c|}
\hline \multicolumn{5}{|l|}{$\begin{array}{l}\text { Mothers } \\
\text { Age (yr) }\end{array}$} \\
\hline Median & 26 & 25 & 26 & 0.72 \\
\hline Interquartile range & $22-29$ & $22-30$ & $22-29$ & \\
\hline Education beyond primary school (\%) & 35.4 & 36.3 & 31.5 & 0.12 \\
\hline Married (\%) & 92.6 & 93.1 & 92.7 & 0.90 \\
\hline Vaginal delivery $(\%)$ & 93.6 & 93.2 & 92.2 & 0.55 \\
\hline Body-mass index $<17(\%)^{\dagger}$ & 0.66 & 0.13 & 0.17 & 0.16 \\
\hline \multicolumn{5}{|l|}{$\mathrm{CD} 4+$ count per $\mathrm{mm}^{3}$} \\
\hline Median & 429 & 440 & 442 & 0.24 \\
\hline Interquartile range & $324-565$ & $330-591$ & $334-586$ & \\
\hline \multicolumn{5}{|l|}{ Hemoglobin (g/dl) } \\
\hline Median & 11 & 11 & 11 & 0.61 \\
\hline Interquartile range & $10-12$ & $10-12$ & $10-12$ & \\
\hline \multicolumn{5}{|l|}{ Infants } \\
\hline Male sex $(\%)$ & 49.7 & 50.0 & 51.1 & 0.86 \\
\hline \multicolumn{5}{|l|}{ Birth weight (kg) } \\
\hline Median & 3.0 & 3.0 & 3.0 & 0.84 \\
\hline Interquartile range & $2.7-3.2$ & $2.7-3.3$ & $2.7-3.3$ & \\
\hline
\end{tabular}

* P values were calculated with the Kruskal-Wallis test for continuous variables and Fisher's exact test for binary variables.

${ }^{t}$ The body-mass index is the weight in kilograms divided by the square of the height in meters. 


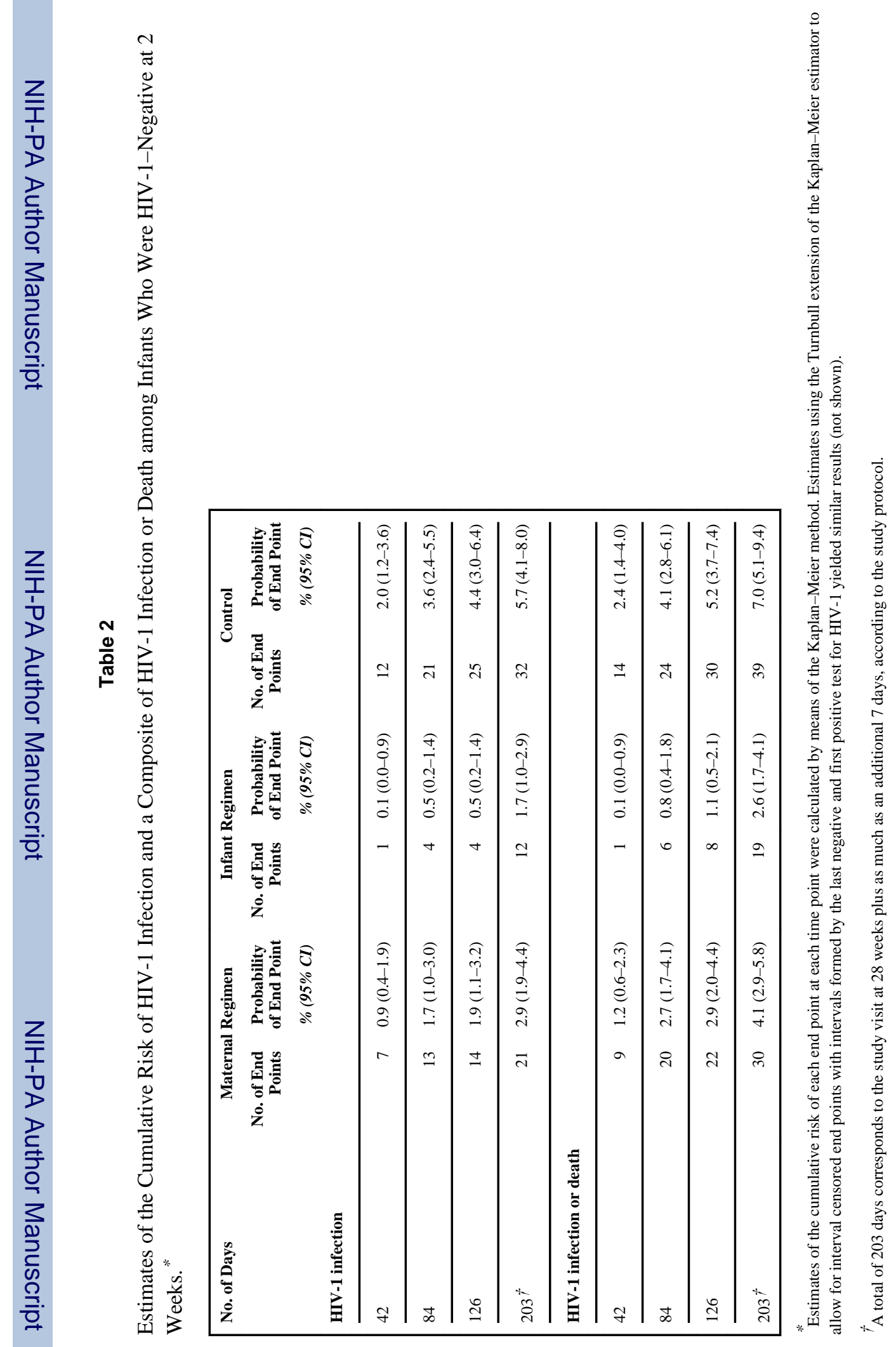

NEngl J Med. Author manuscript; available in PMC 2012 September 13. 


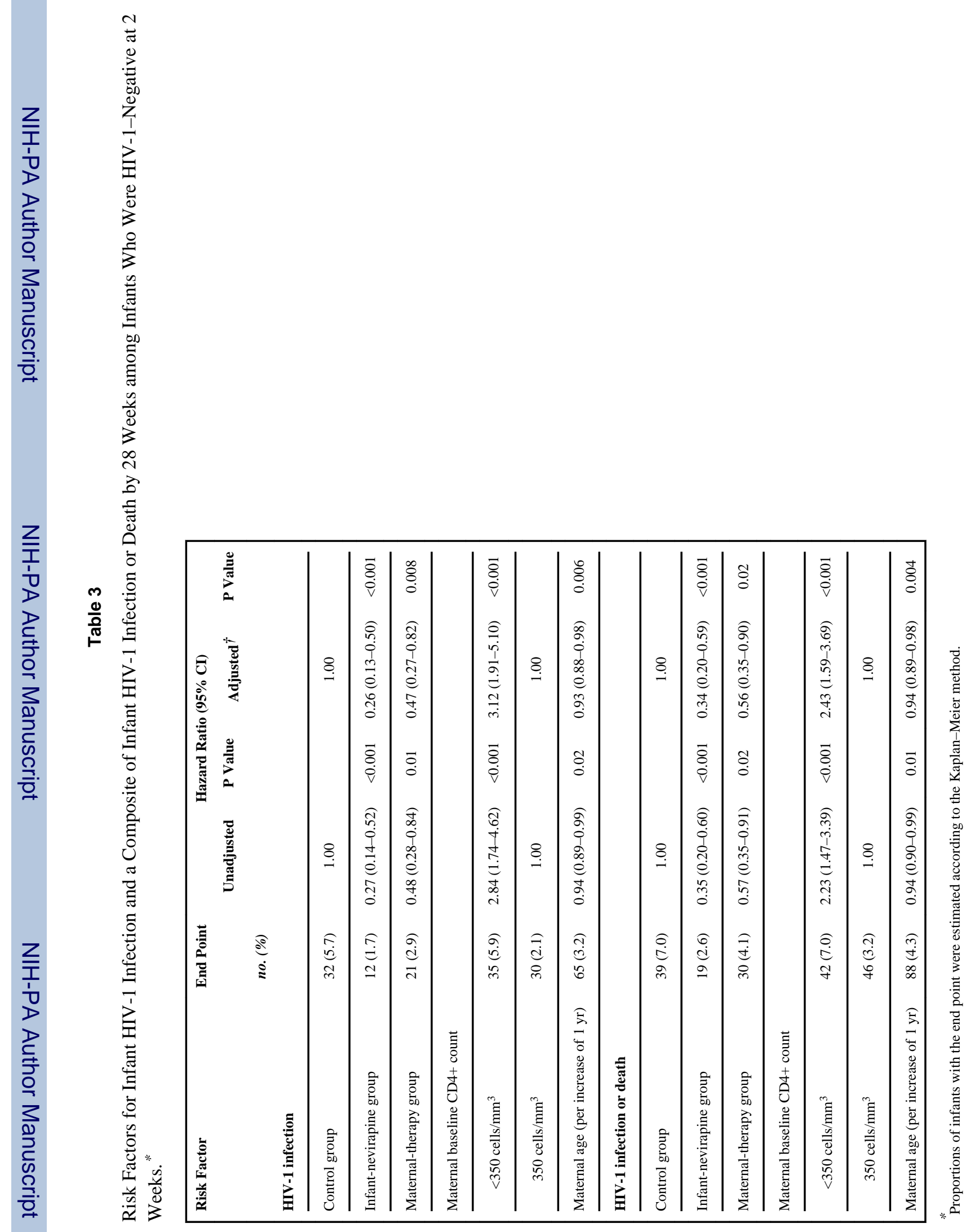

NEngl J Med. Author manuscript; available in PMC 2012 September 13. 
Table 4

Grade 3 or 4 Adverse Events among Mothers and Infants by 36 Weeks. ${ }^{*}$

\begin{tabular}{|c|c|c|c|}
\hline Adverse Event* & $\begin{array}{l}\text { Maternal Regimen } \\
(\mathbf{N}=849)\end{array}$ & $\begin{array}{l}\text { Infant Regimen } \\
(\mathbf{N}=\mathbf{8 5 2})\end{array}$ & $\begin{array}{l}\text { Control } \\
(\mathbf{N}=668)\end{array}$ \\
\hline \multicolumn{4}{|l|}{ Mothers } \\
\hline ALT $25 \times$ upper limit of normal range (\%) & 1.6 & 0.4 & 0.5 \\
\hline Hemoglobin <7.5 g/dl (\%) & 1.6 & 0.8 & 1.7 \\
\hline Neutrophil count $<750$ cells $/ \mathrm{mm}^{3}(\%)^{\dagger}$ & 6.2 & 2.6 & 2.3 \\
\hline Platelet count $<50,000 / \mathrm{mm}^{3}(\%)$ & 1.2 & 0.9 & 1.0 \\
\hline Nevirapine hypersensitivity (no.) ${ }^{\frac{t^{t}}{t}}$ & 6 & 0 & 0 \\
\hline \multicolumn{4}{|l|}{ Infants } \\
\hline ALT $25 \times$ upper limit of normal range (\%) & 0.4 & 0.6 & 0.8 \\
\hline Reduced hemoglobin $(\%)^{\xi}$ & 19.6 & 21.0 & 20.0 \\
\hline Neutrophil count $<400$ cells $/ \mathrm{mm}^{3}(\%)$ & 0.5 & 1.1 & 0.7 \\
\hline Platelet count $<50,000 / \mathrm{mm}^{3}(\%)$ & 1.1 & 1.0 & 1.5 \\
\hline Nevirapine hypersensitivity (no.) & ${ }_{1}$ II & 16 & 0 \\
\hline
\end{tabular}

*

ALT denotes alanine aminotransferase.

${ }_{\mathrm{P}}^{t}<0.001$ for the comparison between the maternal-regimen group and the control group. No other comparisons between either of the intervention groups and the control group were significant.

F In

In mothers, a nevirapine hypersensitivity reaction was defined as rash with one or more of the following: fever, hepatitis, arthralgias, or myalgias during the first 6 to 8 weeks of nevirapine therapy. Severe Stevens-Johnson syndrome developed in two of the six women with hypersensitivity reactions.

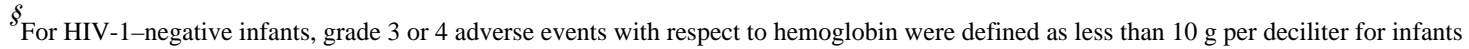
younger than 21 days, less than $8 \mathrm{~g}$ per deciliter for infants between the ages of 22 and 35 days, less than $7 \mathrm{~g}$ per deciliter for infants between the ages of 36 and 56 days, and less than $9 \mathrm{~g}$ per deciliter for infants older than 56 days.

II In infants, a nevirapine hypersensitivity reaction was defined as rash alone of any grade or rash with eosinophilia, fever, or liver involvement and was determined to be associated with nevirapine after the exclusion of other possible causes. The mother of this infant was receiving a nevirapinebased regimen. 